\title{
The Efficiency of Thermal Neutron Detection and Collimation with Microchannel Plates of Square and Circular Geometry
}

\author{
Anton S. Tremsin, W. Bruce Feller, R. Gregory Downing, David F. R. Mildner
}

\begin{abstract}
Detectors with Microchannel Plates (MCPs) are currently widely used in photon and charged particle detection with high spatial $(\sim 10 \mu \mathrm{m})$ and temporal $(<0.5 \mathrm{~ns})$ resolution. All the advances in MCP detection technologies can be successfully implemented for the detection of thermal neutrons by using microchannel plates manufactured from a modified glass mixture doped with neutron absorbing atoms. In this paper we compare the efficiency of thermal neutron detection for two standard MCP geometries: circular-pore and square-pore microchannel plates doped with the ${ }^{10} B$ isotope. The results of our modeling indicate that the detection of thermal neutrons with a square-pore MCP is $11-23 \%$ more efficient than for the circular geometry, and can be as high as $\sim 80 \%$ for the existing MCP technology.

The same microchannel plates can be used as very efficient and compact thermal neutron collimators. In this paper we compare the efficiency of circular- and square-pore MCP collimators with the help of our model, the validity of which has already been verified by our experimental measurements reported last year. The rocking curve of $5 \mathrm{~mm}$ and $2.5 \mathrm{~mm}$ thick MCPs doped with 3 mole \% of ${ }^{\text {nat }} \mathrm{Gd}_{2} \mathrm{O}_{3}$ is predicted to be only $\pm 0.1^{\circ}$ and $\pm 0.3^{\circ}$ wide, respectively, for both geometries.

A very compact device with high thermal neutron detection efficiency and angular sensitivity can be built by combining an MCP neutron detector with an MCP collimator.
\end{abstract}

Index Terms - Neutron detection, neutron optics, position sensitive detectors, high spatial resolution.

\section{INTRODUCTION}

$\mathrm{N}_{\mathrm{r}:}^{\mathrm{o}}$ ON-destructive testing (NDT) based on thermal neutron radiography has a number of advantages over other widely used NDT techniques, such as X-ray and gamma-ray radiography, for example. These different testing methods frequently provide complementary information since the interactions of neutrons and photons with the matter are quite different. Photons interact with the atomic electrons, while

Manuscript received October 12, 2004.

A. S. Tremsin is with the Space Sciences Laboratory, UC Berkeley, Berkeley, CA 94720 USA (e-mail: ast@ssl.berkeley.edu).

W. B. Feller is with NOVA Scientific, Inc. 660 Main St. P.O. Box 928 Sturbridge, MA 01566 USA (e-mail: bfeller@novascientific.com).

R. G. Downing is with NOVA Scientific, Inc. 660 Main St. P.O. Box 928 Sturbridge, MA 01566 USA (e-mail: rgd@ix.netcom.com).

D. F. R. Mildner is with the National Institute of Standards and Technology, Gaithersburg, MD 20899 USA (e-mail: mildner@nist.gov). neutrons interact with the atomic nucleus itself. The contrast in resulting images in X-ray absorption radiography, which is still the most widely used radiographic technique, is thus determined by the difference in the material density between the structures to be imaged and the surrounding media. The absorption coefficient of thermal neutrons is quite different from and unrelated to that of X-ray photons. Neutrons can penetrate high density materials, which are opaque to X-rays, thus allowing the inspection of objects obstructed by a dense material. Furthermore, the attenuation of thermal neutrons (relative to X-rays) is pronounced for lighter elements, such as hydrogen, carbon and their compounds that comprise the base for many organic materials. The few nuclides that are useful for thermal neutron detection include ${ }^{3} \mathrm{He},{ }^{6} \mathrm{Li},{ }^{10} \mathrm{~B},{ }^{113} \mathrm{Cd}$, ${ }^{155} \mathrm{Gd}$ and ${ }^{157} \mathrm{Gd}$ and a few others. Most neutron detection techniques rely on the detection of the energetic particles and photons emitted upon the absorption of neutrons by these nuclides. There exists quite a variety of neutron detection techniques [1]. These include solid state converter films and gas-filled converters, which upon neutron absorption emit photons or charged particles that are subsequently detected by a readout device, such as charge coupled devices (CCDs) [2],[3], foil activation followed by the post-processing readout with imaging plates [4]; scintillating fibers or plates [5]; and superheated liquid detectors [6] and a few others [7].

In this paper we discuss the efficiency of a new type of thermal neutron detector based on microchannel plates (MCPs) doped with ${ }^{10} \mathrm{~B}$ atoms for effective neutron detection [8]-[11]. We compare the efficiency of neutron detection for two standard MCP geometries: hexagonally packed circular pores and square pore microchannel plates doped with ${ }^{10} \mathrm{~B}$ isotope, which has high cross section of thermal neutron capture of 3837 barn for $0.025 \mathrm{eV}$ neutrons.

The same microchannel plates doped with neutron absorbing atoms can be used as very efficient and compact thermal neutron collimators [12]. In section III we compare the efficiency of neutron collimation for circular and square pore geometries. And, finally, in Section IV we discuss the possibility to build an efficient thermal neutron detector with high angular selectivity by combining an MCP detector and an MCP collimator. 


\section{Thermal NEUTRON DEteCtion}

Detectors with microchannel plates are currently widely used in photon and charged particle detection with high spatial $(\sim 10 \mu \mathrm{m})$ and temporal $(<0.5 \mathrm{~ns})$ resolution [13],[14]. All the advances in MCP detection technologies can be successfully applied to the detection of thermal neutrons with microchannel plates manufactured from a modified glass mixture, doped with ${ }^{10} \mathrm{~B}$ neutron absorbing atoms The neutron capture reaction ${ }^{10} \mathrm{~B}(\mathrm{n}, \alpha){ }^{7} \mathrm{Li}$ takes place within the bulk of ${ }^{10} \mathrm{~B}$-doped $\mathrm{MCP}$ glass. Subsequently some alpha and/or ${ }^{7} \mathrm{Li}$ particles escape from the bulk into the adjacent MCP pores, impinge on the secondary electron emissive surface of the MCP pore walls, releasing a number of secondary electrons (Fig. 1). Once these electrons are created by the products of neutron capture, the subsequent processes of signal detection are well developed for UV imaging applications with microchannel plates. For the current neutron-sensitive MCP glass compositions that we have developed the escape ranges of alpha and ${ }^{7} \mathrm{Li}$ particles are approximately $3.5 \mu \mathrm{m}$ and $1.9 \mu \mathrm{m}$, respectively [11].

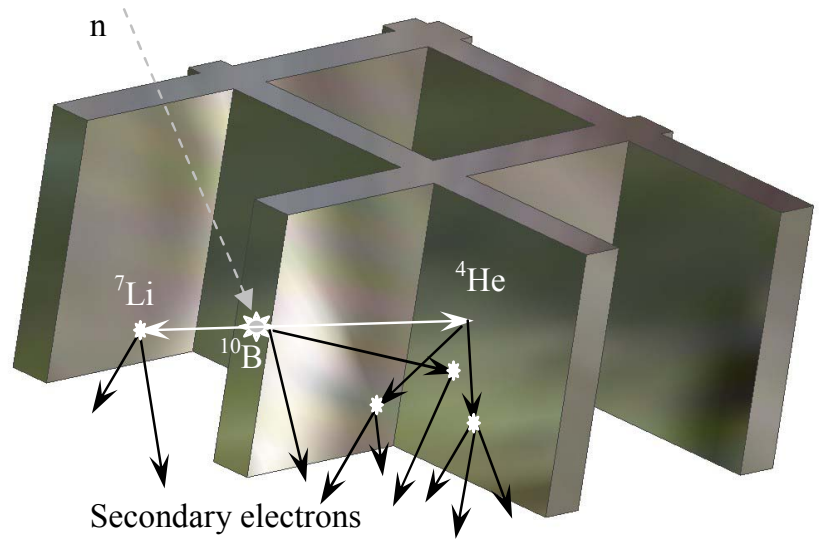

Fig. 1. Schematic diagram of neutron detection within a boron-doped MCP structure (square-pore geometry). Neutron absorption is within the MCP glass by ${ }^{10} \mathrm{~B}$ capture. The reaction products ${ }^{4} \mathrm{He}$ and ${ }^{7} \mathrm{Li}$ escaping to adjacent pores initiate an electron avalanche.

The detection efficiency of an MCP detector can be expressed as a product of three probabilities: $P_{1}-$ the probability of neutron absorption within the MCP glass, $\mathrm{P}_{2}-$ the probability that at least one of the products of neutron capture escapes into an adjacent $\mathrm{MCP}$ pore, and $\mathrm{P}_{3}-$ the probability that an electron avalanche is formed within that pore. Obviously the geometry of a particular microchannel plate has a strong effect on probabilities $\mathrm{P}_{1}$ and $\mathrm{P}_{2}$. Our detailed modeling of thermal neutron detection efficiency with square pore MCPs [11] indicates that the efficiency can be as high as $\sim 80 \%$ for the existing MCP technology. In this paper we compare two standard MCP geometries: square-pore and hexagonally packed circular-pore MCPs. We will give the detailed description of the circular pore geometry calculations in a future paper.

Microchannel plates typically consist of several million microchannels fused together into a monolithic disk-like structure. The pores are usually of a circular or square geometry (Fig. 2). The typical width of the pore varies between 6-15 $\mu \mathrm{m}$ and the wall thickness is usually $2-3 \mu \mathrm{m}$. The thickness of an electron-multiplying MCP is typically 40250 times larger than the pore diameters (equivalent to 0.24$3.75 \mathrm{~mm}$ ), and a practical MCP can have an input area as large as $10 \times 10 \mathrm{~cm}^{2}$.
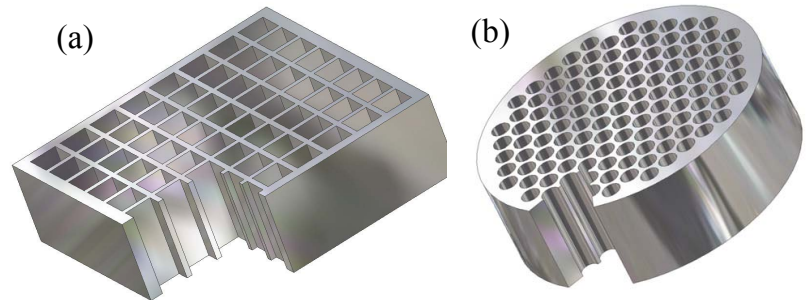

Fig. 2. A section of a square (a) and circular (b) pore microchannel plates (drawn not to scale).

The relative simplicity of manufacturing an MCP with circular pores is accompanied by the apparent reduction of the probability $\mathrm{P}_{2}$ relative to the square pore geometry because the distance from the point of neutron capture to adjacent pores is on average larger. That reduction in detection efficiency has to be estimated for the applications where the detection efficiency is crucial.

\section{A. $P_{1}$ - probability of neutron absorption}

Fig. 3 shows the probability of neutron absorption $\mathrm{P}_{1}$ for typical square- and circular-pore MCPs with identical pore widths and center-to-center spacing as a function of MCP thickness. The absorption probability $\mathrm{P}_{1}$ is obviously larger for the circular pore geometry due to a larger amount of MCP glass in a circular pore structure.

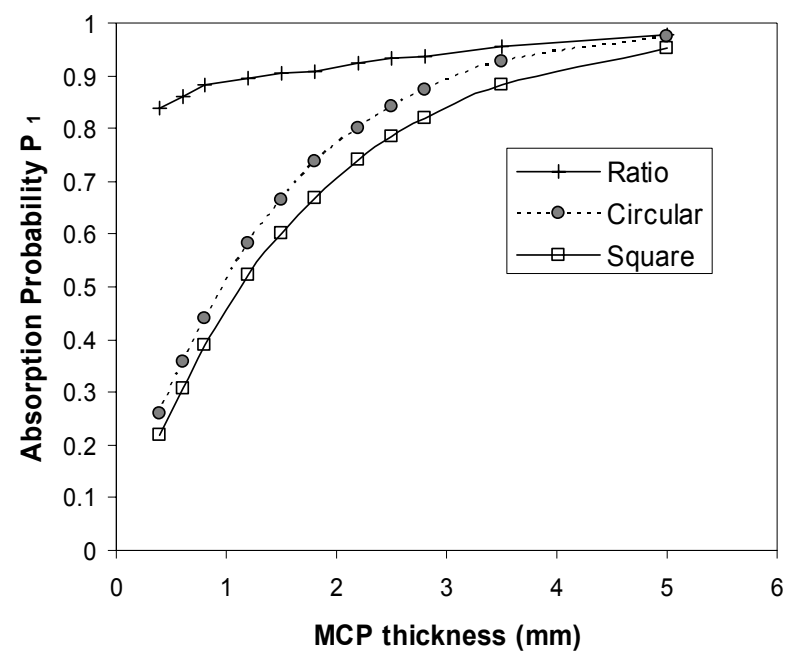

Fig. 3. The probability $\mathrm{P}_{1}$ of thermal neutron absorption within the ${ }^{10} \mathrm{~B}$-doped MCP glass as a function of MCP thickness for square- and circular-pore geometry. The MCP has $10 \mu \mathrm{m}$ pores on centers $12 \mu \mathrm{m}$ apart, doped with 10 mole $\%$ of ${ }^{10} \mathrm{~B}_{2} \mathrm{O}_{3}$ and has $10^{\circ}$ pore bias. Neutrons are incident normal to the MCP surface.

The deficiency of neutron absorption in case of square pores can be compensated by manufacturing a thicker MCP or by 
stacking several MCPs or even entire detectors (as described in ref. [11]). The absorption probability $\mathrm{P}_{1}$ is above $86 \%$ for both square- and circular-pore microchannel plates (or a stack of them) thicker than $3.2 \mathrm{~mm}$. For $10 \mu \mathrm{m}$ pore $\mathrm{MCP}$ that corresponds to length to pore diameter ratio of $L / D=320: 1$. Currently MCPs are produced with L/D ratios of up to 250:1. Therefore a stack of two 160:1 MCPs will have $86 \%$ absorption probability, and a stack of three 160:1 MCPs will absorb $95 \%$ of incoming neutrons.
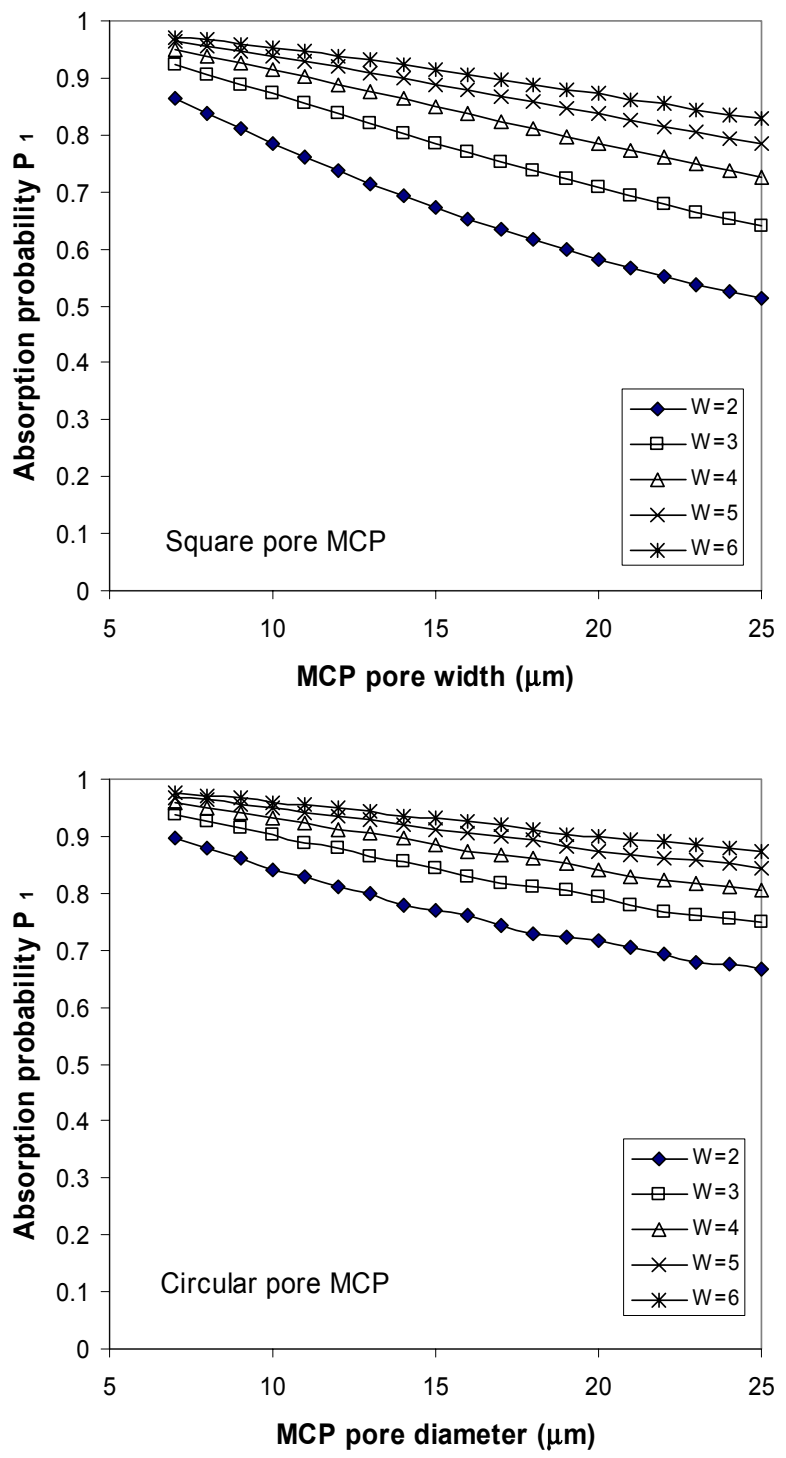

Fig. 4. The probability $P_{1}$ of thermal neutron absorption within the ${ }^{10} \mathrm{~B}$-doped MCP glass as a function of MCP pore width/diameter, with fixed value of MCP wall thickness $\mathrm{W}$, shown in $\mu \mathrm{m}$ in the legend. The $2.5 \mathrm{~mm}$ thick MCP is doped with 10 mole $\%$ of ${ }^{10} \mathrm{~B}_{2} \mathrm{O}_{3}$ and has $10^{\circ}$ pore bias. Neutrons are incident normal to the MCP surface.

Fig. 4 shows the predicted absorption probability $\mathrm{P}_{1}$ as a function of pore width/diameter when the wall thickness is fixed. The aspect ratio of modeled MCPs was fixed at the value of $L / D=250: 1$, the largest aspect ratio achievable with current manufacturing technology. With the help of our absorption probability model (already verified by experimental measurements [12]) we can select a proper thickness of an MCP stack for a given set of geometrical parameters and required absorption efficiency $\mathrm{P}_{1}$.

\section{B. $P_{2}$ - probability of ${ }^{4} \mathrm{He}$ and/or ${ }^{7}$ Li escape into adjacent MCP pores}

The escape range of alpha and ${ }^{7} \mathrm{Li}$ particles matches well the current thickness of microchannel plate walls. Obviously the thinner the walls are the higher is the escape probability $\mathrm{P}_{2}$, but the probability $\mathrm{P}_{1}$ gets smaller at the same time. As mentioned earlier $\mathrm{P}_{1}$ can be increased by stacking a few MCPs, but there is a limit to how many of them can be stacked in a practical detection device. One of the limiting factors is the high voltage required for the stack operation (the thicker the stack, the larger is the voltage). Besides, the mechanical stability of MCPs with thin walls can impose a problem. Thus the wall thickness of neutron detecting MCP has to be optimized in order to achieve the best detection efficiency.

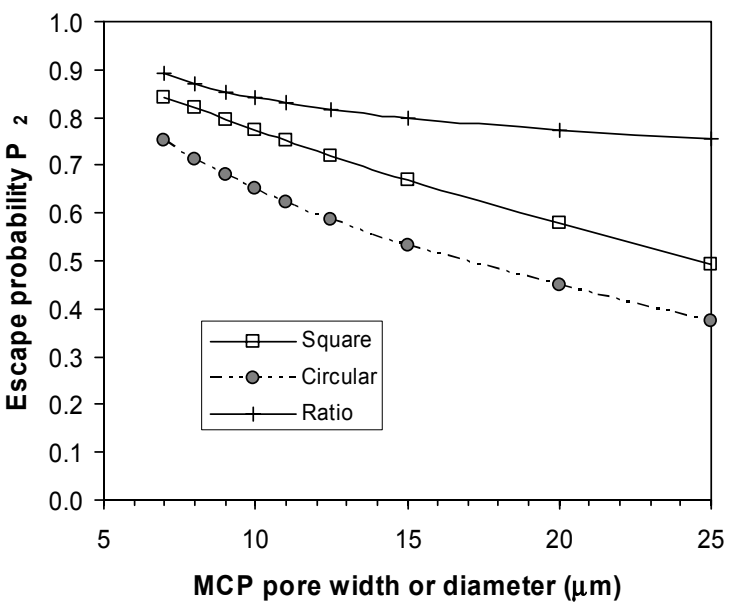

Fig. 5. The probability that the products of ${ }^{10} \mathrm{~B}$ neutron capture escape into an adjacent MCP pore as a function of the MCP pore width/diameter D for circular and square pore geometry. Ratio of $\mathrm{D} / \mathrm{W}$ fixed at $5: 1$.

A detailed description of our model of neutron detection with square pore MCP can be found in ref. [11] and circular pore geometry description is in preparation. The values of escape probability $\mathrm{P}_{2}$ predicted with the help of the model are shown in Fig. 5 for both square and circular pore geometry. The probability $\mathrm{P}_{2}$ is plotted as a function of MCP pore diameter (in case of circular pores) or width (in case of square pores). The wall thickness for these curves is $1 / 5$ of pore width/diameter, as it is in Fig. 3 (e.g. $10 \mu \mathrm{m}$ pores, $2 \mu \mathrm{m}$ walls). Most current MCPs are produced with such ratio between pore and wall widths. The escape probability $\mathrm{P}_{2}$ of a square pore MCP is better by $11 \%$ for the pores of $7 \mu \mathrm{m}$, by $15.6 \%$ for $10 \mu \mathrm{m}$ pores and by $20.2 \%$ for $15 \mu \mathrm{m}$ pores. The escape probability values $\mathrm{P}_{2}$ can be as high as 0.84 (square- 
pore) and 0.75 (circular-pore) for $7 \mu \mathrm{m}$ pore microchannel plate.

Fig. 6 shows the predicted escape probability $P_{2}$ as a function of pore width/diameter when the wall thickness (rather than the ratio of the pore and the wall width) is fixed (as in Fig. 4). For a fixed value of wall thickness, the probability of escape $\mathrm{P}_{2}$ monotonically increases with the pore width in case of square geometry. For circular pores $\mathrm{P}_{2}$ is not a monotonic function and $\mathrm{P}_{2}$ decreases with the pore diameter increase above some optimal value for a given wall thickness.
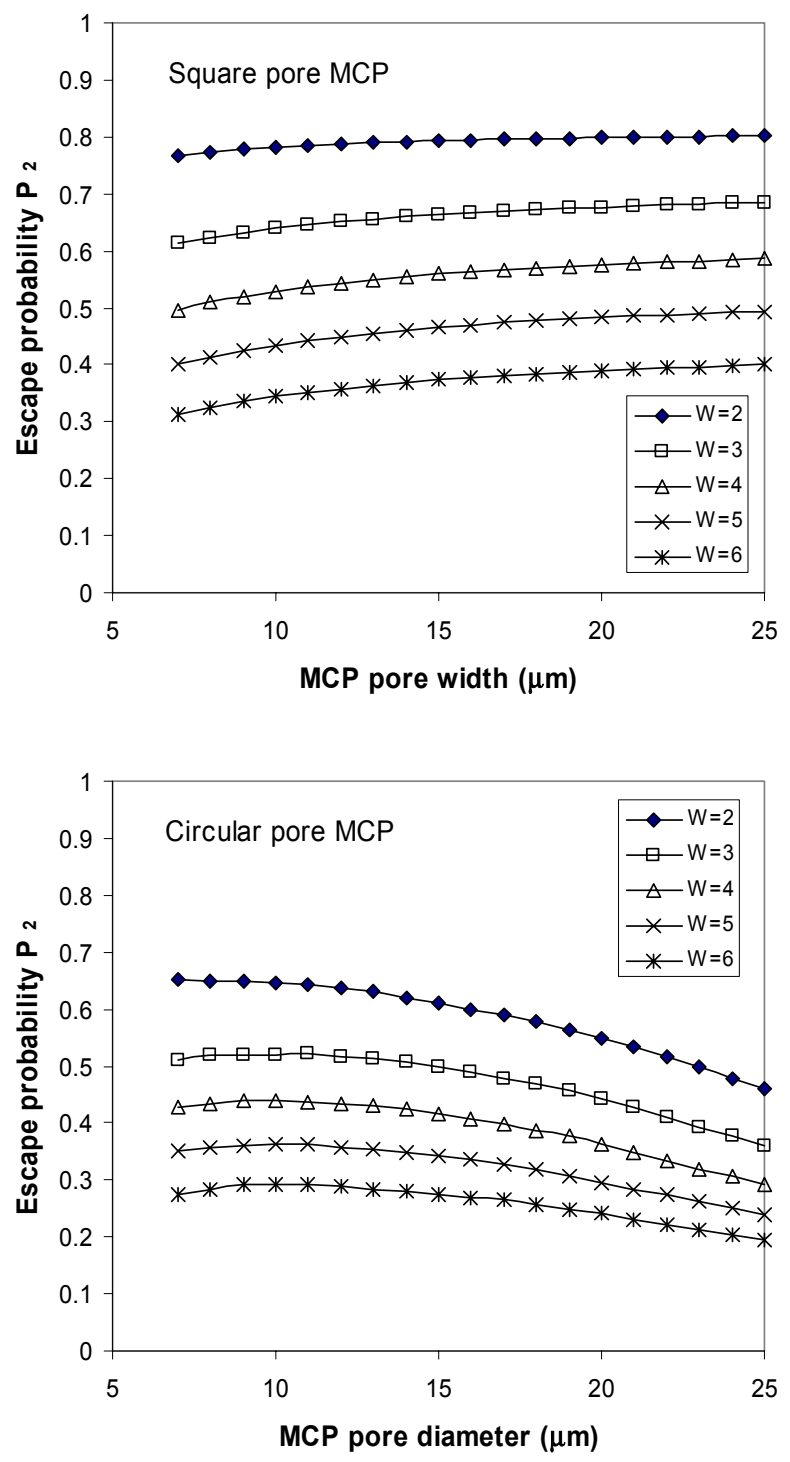

Fig. 6. The probability $\mathrm{P}_{2}$ that at least one of the neutron capture products $\left({ }^{4} \mathrm{He}\right.$ or ${ }^{7} \mathrm{Li}$ ) escapes into an adjacent $\mathrm{MCP}$ pore as a function of $\mathrm{MCP}$ pore width/diameter, with fixed value of MCP wall thickness $\mathrm{W}$, shown in $\mu \mathrm{m}$ in the legend.

Our modeling results indicate that the neutron detection efficiency $\mathrm{P}_{\text {neutron }}=\mathrm{P}_{1} \mathrm{xP}_{2} \mathrm{xP}_{3}$ is better for the MCPs with square pores. Their inferior neutron absorption $\mathrm{P}_{1}$ can be compensated by the increase of MCP thickness, while the lower probability $\mathrm{P}_{2}$ for the circular geometry cannot be compensated. The probability $\mathrm{P}_{3}$ is close to unity for both types of MCP geometry [11]. Therefore, we conclude that the thermal neutron detection efficiency of circular-pore microchannel plates is in general lower than that of a similar square pore MCP by as much as the difference in $\mathrm{P}_{2}$, i.e. 11-23 $\%$ (Fig. 5).

\section{THERMAL NEUTRON COLLIMATION}

The same doped microchannel plates can also be used for thermal neutron collimation, as shown in our recent paper [12]. The predicted results presented there agree well with our first experimental measurements, proving the validity of our model in terms of the absorption probability $\mathrm{P}_{1}$. Our model predicts that for the existing manufacturing limits on the doping level in the MCP glass a collimator doped with ${ }^{157} \mathrm{Gd}$ atoms is more efficient than one doped with ${ }^{10} \mathrm{~B}$ [12], while MCPs with ${ }^{10} \mathrm{~B}$ doping are more suitable for neutron detection. This is because the detection of the low energy conversion electron and gamma ray emissions from the ${ }^{157} \mathrm{Gd}(\mathrm{n}, \gamma){ }^{158} \mathrm{Gd}$ reaction is difficult to discriminate from background events in the detector, especially in the case of operation in a high radiation field. MCP collimators do not need the electron multiplication properties and therefore several MCP manufacturing processes can be eliminated. Thus, we believe that although the same ${ }^{10} \mathrm{~B}$-doped MCP can be used for either thermal neutron detection or collimation, more efficient collimators can be built with ${ }^{157} \mathrm{Gd}$ doped microchannel plates.

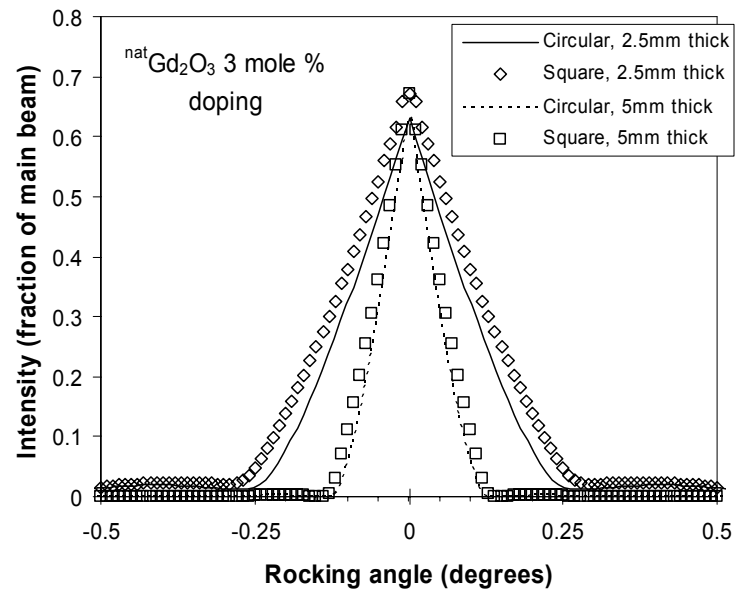

Fig. 7. The predicted rocking curves for square- (symbols) and circular-pore (curves) MCP collimators for thermal neutrons. MCPs are doped with 3 mole $\%$ of ${ }^{\text {nat }} \mathrm{Gd}_{2} \mathrm{O}_{3}$, and pores are $10 \mu \mathrm{m}$ wide on $12 \mu \mathrm{m}$ centers. The MCP thickness is shown in the legend.

Fig. 7 shows two rocking curves for a typical circular- and square-pore microchannel plates that can be built with the existing technology. Collimation within $\pm 0.25^{\circ}$ can be achieved with $10 \mu \mathrm{m}$ pore MCP with the available aspect ratio of 250:1. The difference between circular and square geometry collimators is not significant and can roughly be characterized by the difference in the open area ratio (the ratio of pore area to the area of entire MCP surface), determining the maximum transmission of the collimator. For a $10 \mu \mathrm{m}$ pore and $2 \mu \mathrm{m}$ 
wall MCP this ratio is 0.69 and 0.63 for square and circular MCPs, respectively. The performance of MCP collimators shown in Fig. 7 is calculated for a parallel beam of neutrons. Most neutron beams have some angular divergence, and therefore these predicted curves should be convolved with the angular profile of a particular beam itself (typically of a Gaussian shape). Small-angle reflection from the pore walls is not taken into account in our model, but our first experimental measurements indicate that the reflection does not have a considerable effect on the collimator performance beyond moderate smoothing of the triangular shape of the rocking curve [12].

\section{Angular SElective Neutron Detection}

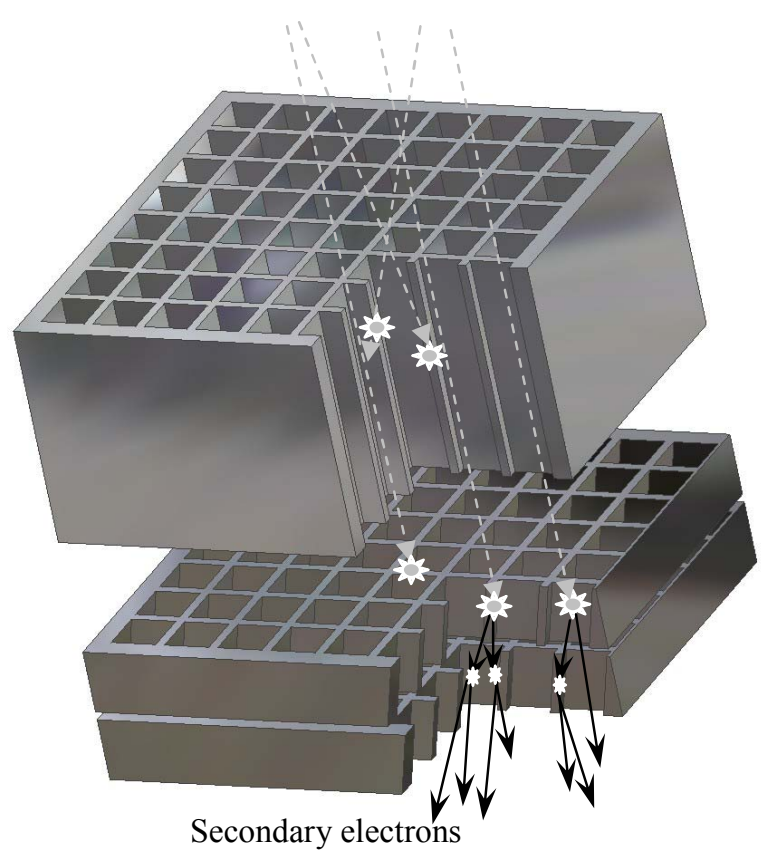

Fig. 8. Schematic diagram of the proposed angular selective $\left( \pm 0.25^{\circ}\right)$ thermal neutron counting detector with high spatial resolution (not shown to scale). The front MCP serves as a collimator, and no voltage is applied to it. The entire device is $<10 \mathrm{~mm}$ thick. Secondary electrons are collected by one of the standard MCP readouts, such as a cross delay line or cross strip anode.

Combining MCP thermal neutron collimators and detectors in a single device should produce a thermal neutron counting detector with high detection efficiency, spatial resolution and angular selectivity, Fig. 8. One attractive feature of such a device is its compactness - less than $10 \mathrm{~mm}$ thick. The angular selectivity of such a device can be switched off by applying voltage to the front $\mathrm{MCP}$, which will produce the secondary electrons to be amplified by the lower MCP stack. By applying a reverse bias to the front $\mathrm{MCP}$ and placing a neutrontransparent electron collecting element in front of the first $\mathrm{MCP}$, one can measure the ratio of "on angle" and "off angle" neutrons. All of this is done by controlling the bias voltages applied to microchannel plates, without any need for mechanical changes.

\section{ACKNOWLEDGMENT}

A.S. Tremsin is grateful to NOVA Scientific, Inc. for the travel financial support.

\section{REFERENCES}

[1] A. J. Peurrung, "Recent developments in neutron detection", Nucl. Instrum. Methods, vol. A 443, pp.400-415, 2000

[2] D. S. McGregor, M. D. Hammig, Y. H. Yang et al., "Design considerations for thin film coated semiconductor thermal neutron detectors - I: basics regarding alpha particle emitting neutron reactive films“, Nucl. Instrum. Methods, vol. A 500, pp. 272-308, 2004.

[3] H. Friedrich, V. Dangendorf, A. Brauning-Demian, "Position-sensitive thermal neutron detector with Li-6-foil converter coupled to wire chambers", Applied Physics A-Materials Science \& Processing. vol. 74, pp. S124-S126, 2002.

[4] K. Takahashi, S. Tazaki, J. Miyahara, Y. Karasawa, N. Niimura, "Imaging performance of imaging plate neutron detectors", Nucl. Instrum. Methods, vol. A 377, pp.119-122 1996.

[5] N. Izumi, R.A. Lerche, T.W. Phillips, G.J. Schmid, M.J. Moran, J.A. Koch, H. Azechi, T.C. Sangster, "Development of a gated scintillation fiber neutron detector for areal density measurements of inertial confinement fusion capsules”, Rev. Sci. Instrum., vol. 74, pp. 1722-1725, 2003.

[6] S. C. Roy, B. Roy, "Use of superheated liquid in neutron detection", Current Science, vol. 84, pp.516-528, 2003.

[7] G.W. Fraser, "Thermal neutron imaging", Proc. SPIE, vol. 2339, International Conference on Neutrons and Their Applications, George Vourvopoulos; Themis Paradellis; Eds., pp. 287-301, 1995.

[8] G.W. Fraser, J.F. Pearson, "The direct detection of thermal-neutrons by imaging microchannel-plate detectors", Nucl. Instrum. Methods, A 293, pp.569-574, 1990.

[9] G.W.Fraser, J.F. Pearson, W.B. Feller, L.M. Cook, O.S. Al-Horayess, "Thermal neutron imaging using microchannel plates", Proc. SPIE, vol. 1737, 298, 1992.

[10] W. B. Feller, R. G. Downing, P. L. White, "Neutron field imaging with microchannel plates", Proc. SPIE, vol. 4141, Hard X-Ray, Gamma-Ray, and Neutron Detector Physics II, pp.291-302, 2000.

[11] A. S. Tremsin, W. B. Feller, R. G. Downing, "Efficiency optimization of neutron imaging detectors with ${ }^{10} \mathrm{~B}$ doped MCPs", in print, Nucl. Instrum. Methods section A 2004.

[12] A. S. Tremsin, D. F. R. Mildner, W. B. Feller, R. G. Downing, "Very Compact High Performance Microchannel Plate Neutron Collimators", IEEE Trans. Nucl. Sci., vol. 51, pp.1020-1024, 2004.

[13] M. Ghioni, S. Cova, C. Samori, F. Zappa, "True constant fraction trigger circuit for picosecond photon-timing with ultrafast microchannel plate photomultipliers", Rev. Sci. Instrum. vol. 68, pp.2228-2237, 1997

[14] A. S. Tremsin, O. H. W. Siegmund, J. V. Vallerga, J. S. Hull, R. Abiad, "Cross strip readouts for photon counting detectors with high spatial and temporal resolution", IEEE Trans. Nucl. Sci., vol. 51, pp.1707-1711, 2004. 